\title{
Sensitive Electrochemical Immunosensor for Detection of Mycotoxins Aflatoxin B1 Using Disposable screen- Printed Carbon Electrode
}

\author{
Ping liu, Xingpu Qi, Huanxin Zhang, Yi Zheng* \\ Jiangsu Agri-animal Husbandry Vocational College, No 8 East Phoenix Road, Taizhou, Jiangsu, \\ 225300, P.R.China \\ *E-mail: qixingpu@ 163.com
}

doi: $10.20964 / 2021.03 .44$

Received: 1 November 2020/ Accepted: 23 December 2020 / Published: 31 January 2021

\begin{abstract}
This study presented the preparation of sensitive electrochemical immunosensor to detect mycotoxins aflatoxin B1 (AFB1) by immobilization of monoclonal antibodies of AFB1 on disposable screenprinted carbon electrode (SPCE) surface (anti-AFB1/SPCE). The morphological and structural properties of prepared SPCE were studied by XRD and FESEM analysis which showed the carbon structure of SPCE covered the electrode surface with rough and porous morphology. Differential pulse voltammetry (DPV) and Cycle voltammetry (CV) techniques were applied to record the electrochemical responses of anti-AFB1/SPCE and SPCE. CV studies showed the electrochemical responses of antiAFB1/SPCE were more stable and sensitive toward SPCE in $\left[\mathrm{Fe}(\mathrm{CN})_{6}\right]^{3-/ 4-}$ as redox probe solution due to the synergistic effect between porous morphology of SPCE and immobilization of anti-AFB1 and its the great electrochemical reaction capability and fast rates of electron transfer on anti-AFB1/SPCE surface. DPV measurements showed the linear range and limit of detection of AFB1 on anti-AFB1/SPCE were obtained 10 to $120 \mathrm{ng} \cdot \mathrm{ml}^{-1}$ and $0.007 \mathrm{ng} \cdot \mathrm{ml}^{-1}$, respectively which illustrating the comparable or better electrochemical performance of anti-AFB1/SPCE for determination of AFB1 in comparison with other reported AFB1 immunosensors in literatures. Studying the capability of anti-AFB1/SPCE in a prepared real sample of maize showed the obtained AFB1 concentration in pure real solution was 9.73 $\mathrm{ng} \mathrm{ml}^{-1}$ that was consistent with the results obtained by high performance liquid chromatography method. Furthermore, results demonstrated the obtained recovery and relative standard deviation values were acceptable and the prepared immunosensor was reliable to determine AFB1 in real samples.
\end{abstract}

Keywords: Immunosensor; Aflatoxin B1; Cycle voltammetry; Differential pulse voltammetry; Linear range; Limit of detection

\section{$\underline{\text { FULL TEXT }}$}


(C) 2021 The Authors. Published by ESG (www.electrochemsci.org). This article is an open access article distributed under the terms and conditions of the Creative Commons Attribution license (http://creativecommons.org/licenses/by/4.0/). 